\title{
NASOANGIOFIBROMA JUVENIL: CONCORDÂNCIA INTEROBSERVADORES NO ESTADIAMENTO POR TOMOGRAFIA COMPUTADORIZADA*
}

\author{
Jefferson Luiz Gusso', Ricardo Pires de Souza ${ }^{2}$, Abrão Rapoport ${ }^{3}$, Aldemir Humberto Soares ${ }^{4}$
}

Resumo Com o advento da tomografia computadorizada, a avaliação dos locais de acometimento e extensão do nasoangiofibroma juvenil tornou-se mais precisa, o que facilitou o correto estadiamento pré-operatório. 0 objetivo deste trabalho é avaliar a concordância interobservadores, por meio da análise por tomografia computadorizada, de 15 casos de nasoangiofibroma juvenil em relação à extensão para locais relacionados com altos índices de recidivas. Todos os pacientes eram do sexo masculino, com idade média de 15,8 anos. A análise da concordância entre os observadores quanto à extensão e envolvimento da fossa infratemporal, seios esfenóide e cavernoso e fossa craniana média foi excelente, em relação à fissura orbitária superior foi boa e em relação à base do processo pterigóide e fossa craniana anterior foi ruim. Concluiu-se que a análise apresenta alta concordância entre os observadores para os locais estabelecidos, com exceção da base do processo pterigóide e da fossa craniana anterior.

Unitermos: Nasoangiofibroma juvenil. Tomografia computadorizada. Concordância interobservadores.

Abstract Juvenile nasopharyngeal angiofibroma: interobserver agreement in cancer staging using computed tomography.

The advent of computed tomography permitted a much more precise evaluation of tumor localization, thus facilitating presurgical staging. The objective of this study was to evaluate the interobserver agreement on the analysis of computed tomography scans of 15 patients with juvenile nasopharyngeal angiofibroma, regarding assessment of tumor extension to sites of high recurrence rate. All patients were male and had a mean age of 15.8 years. The results of the interobserver agreement analysis regarding tumor invasion and extension to the infratemporal fossa, cavernous sinus, sphenoid sinus and medium cranial fossa were excellent. Identification of involvement of the superior orbital fissure was considered good. Interobserver agreement regarding tumor extension to the base of pterigoid process and anterior cranial fossa was considered poor. We concluded that there is a very good interobserver agreement on the evaluation of the established localization of tumor invasion, except when evaluating the base of the pterigoid process and the anterior cranial fossa.

Key words: Juvenile nasopharyngeal angiofibroma. Computed tomography. Interobserver agreement.

\section{INTRODUÇÃO}

$\mathrm{O}$ nasoangiofibroma juvenil (NAFJ) é um tumor vascular histologicamente benigno, com comportamento biológico agressivo, que afeta jovens do sexo masculino. Apresenta curso potencialmente maligno, por causa do seu crescimento

\footnotetext{
* Trabalho realizado no Complexo Hospitalar Heliópolis, São Paulo, SP.

1. Médico Radiologista, Mestre em Medicina pelo Serviço de Cirurgia de Cabeça e Pescoço do Complexo Hospitalar Heliópolis.

2. Médico Radiologista, Doutor em Medicina pela Universidade de São Paulo, Diretor do Serviço de Diagnóstico por Imagem do Complexo Hospitalar Heliópolis.

3. Livre-Docente, Cirurgião de Cabeça e Pescoço, Coordenador do Curso de Pós-graduação em Cirurgia de Cabeça e Pescoço do Complexo Hospitalar Heliópolis.

4. Médico Radiologista, Presidente do Colégio Brasileiro de Radiologia.

Endereço para correspondência: Dr. Jefferson Luiz Gusso. Rua Pedro Ivo, 318, Centro. Curitiba, PR, 80010-020. E-mail: jlgusso@uol.com.br

Aceito para publicação em 31/5/2001.
}

invasivo e extensão para regiões adjacentes, estando associado a elevada morbidade e ocasional mortalidade ${ }^{(1,2)}$.

O NAFJ é uma neoplasia não-encapsulada, classicamente composta por rica rede vascular de permeio a estroma fibro$\mathrm{so}^{(3)}$. O seu suprimento arterial provém preferencialmente da artéria carótida externa, principalmente da artéria maxilar interna $^{(4)}$. Origina-se na fossa pterigopalatina, na margem superior do forame esfenopalatino ${ }^{(5,6)}$, e apresenta uma prevalência de $0,05 \%$ dos tumores de cabeça e pescoço ${ }^{(7)}$.

A causa deste tumor permanece desconhecida, no entanto, acredita-se que sofra influência hormonal, pelo fato de apenas acometer indivíduos jovens do sexo masculino ${ }^{(8-10)}$.

A avaliação do NAFJ sempre foi um desafio para os cirurgiões. Até bem pou- co tempo, existiam poucos métodos capazes de auxiliar a história clínica e o exame físico. Restritos basicamente à radiografia convencional e angiografia, os cirurgiões, muitas vezes, realizavam tratamentos baseados em suposições e história natural deste tumor.

$\mathrm{O}$ advento de novos métodos de imagem, sobretudo a tomografia computadorizada (TC), possibilitou uma melhor avaliação dos tecidos profundos do pescoço em relação ao reconhecimento da extensão tumoral, começando uma nova era no tratamento dessas lesões, no que diz respeito ao planejamento cirúrgico, diminuição das complicações e das reci$\operatorname{divas}^{(11,12)}$.

A avaliação correta pela TC é fator primário na avaliação pré-operatória dos NAFJs. Reconhecendo-se esta importância, este trabalho se propõe analisar a 
concordância interobservadores na definição de extensões tumorais, relacionadas com altos índices de recidivas ${ }^{(13)}$.

\section{Objetivo}

Avaliar a concordância entre três observadores, pela análise por TC, em relação ao envolvimento de locais específicos da base do crânio pelo NAFJ, ou seja: fossa infratemporal, fissura orbitária superior, seio esfenoidal, seio cavernoso, base do processo pterigóide, fossa craniana anterior e fossa craniana média.

\section{CASUÍSTICA E MÉTODO}

Foram revisados, retrospectivamente, os filmes tomográficos de 15 pacientes com NAFJ, confirmados histologicamente pelo Serviço de Anatomia Patológica do Hospital Heliópolis, num período de 11 anos (de janeiro de 1985 a maio de 1996). Todos os pacientes eram do sexo masculino, com idade variando de 12 a 20 anos e média de 15,8 anos.

Para a inclusão neste trabalho os pacientes deveriam ser tratados no Serviço de Cirurgia de Cabeça e Pescoço do Hospital Heliópolis, apresentar confirmação anatomopatológica, não ter recebido tratamento prévio ou qualquer procedimento intervencionista antes da realização das imagens tomográficas.

Foram excluídos deste trabalho os pacientes que apresentavam tumores recidivados, exames realizados com técnica inadequada, isto é, as imagens deveriam conter os planos axiais e coronais após a administração do meio de contraste iodado endovenoso.

Para se avaliar a concordância interobservadores no que se refere à invasão dos locais pré-definidos, todos os casos foram analisados separadamente por três radiologistas com experiência em tumores de cabeça e pescoço, mediante questionário previamente estabelecido. Os observadores respondiam a um questionário direto, com respostas simples ( ou não), no qual constavam os locais previamente definidos.

Todas as imagens tomográficas foram realizadas em aparelhos de terceira geração, com espessura de corte e incremento de mesa de 3 e $5 \mathrm{~mm}$. As imagens no plano coronal foram posicionadas perpendiculares à lâmina horizontal do etmóide e no plano axial paralelas ao palato duro. Administrou-se cerca de 80 a $120 \mathrm{ml}$ de contraste endovenoso previamente às imagens axiais.

Para a avaliação da concordância interobservadores na leitura dos exames, foi utilizado o índice kappa, com os respectivos critérios de concordância.

\section{RESULTADOS}

Realizou-se análise conjunta dos três observadores (radiologistas) testando-se a hipótese nula de inexistência de concordância entre os avaliadores (kappa = 0) versus a hipótese alternativa de existência de concordância (kappa $>0$ ). Para tanto, estimou-se o coeficiente de correlação de kappa, levando-se em conta as avaliações dos três radiologistas. $\mathrm{Na} \mathrm{Ta}-$ bela 1 são apresentados os resultados estimados, juntamente com o intervalo de 95\% de confiança para o coeficiente de correlação de kappa, o valor de $p$ associado ao teste e a classificação associada à concordância estimada.

Para a fossa infratemporal e seio esfenoidal, estimou-se o coeficiente de correlação de kappa igual a 1,00, indicando isto excelente concordância entre as avaliações para estes locais, com intervalo de confiança de $95 \%$ variando de $(0,71$; $1,00)$, sendo este dado significativo.

Para a fissura orbitária superior, estimou-se o coeficiente de correlação de kappa igual a 0,62 , indicando isto boa concordância entre as avaliações para este local, com intervalo de confiança de $95 \%$ variando de $(0,33 ; 0,91)$, sendo este dado significativo.

Para o seio cavernoso e fossa craniana média, estimou-se o coeficiente de correlação de kappa igual a 0,82 e 0,81, respectivamente, indicando isto uma excelente concordância entre as avaliações para estes locais, com intervalos de confiança de $95 \%$ variando de $(0,53 ; 1,00)$ para o seio cavernoso e $(0,52 ; 1,00)$ para a fossa craniana média, sendo estes dados significativos.

Para a base do processo pterigóide e fossa craniana anterior, estimou-se o coeficiente de correlação de kappa igual a 0,18 e 0,10 , respectivamente, indicando isto uma concordância ruim entre as avaliações para estes locais, com intervalos de confiança de $95 \%$ variando de $(0,00 ; 0,47)$ para a base do processo pterigóide e $(0,00 ; 0,39)$ para a fossa craniana anterior.

\section{DISCUSS̃̃o}

O NAFJ é neoplasia benigna rara, que apresenta achados distintos e característicos. É um tumor que, caracteristicamente, afeta pacientes do sexo masculino na adolescência e apresenta localização e crescimento constante, sendo sua extensão bem reconhecida. A predileção por crescimento através de linhas de menor resistência, forames e fissuras naturais faz com que este tumor seja previsível em suas extensões ${ }^{(14)}$.

Desde a década de 70 o NAFJ tem sido melhor estudado quanto ao seu tamanho

Tabela 1 Índice de concordância estimado pelo método kappa entre os três observadores.

\begin{tabular}{|l|c|c|c|l|}
\hline \multicolumn{1}{|c|}{ Local } & Kappa estimado & $\begin{array}{c}\text { Limites de } 95 \% \text { de } \\
\text { confiança }\end{array}$ & Valor de $p$ & Classificação \\
\hline FIT & 1,00 & $(0,71 ; 1,00)$ & $<0,0001$ & Excelente \\
SE & 1,00 & $(0,71 ; 1,00)$ & $<0,0001$ & Excelente \\
FOS & 0,62 & $(0,33 ; 0,91)$ & $<0,0001$ & Bom \\
SC & 0,82 & $(0,53 ; 1,00)$ & $<0,0001$ & Excelente \\
FCM & 0,81 & $(0,52 ; 1,00)$ & $<0,0001$ & Excelente \\
BPP & 0,18 & $(0,00 ; 0,47)$ & 0,1178 & Ruim \\
FCA & 0,10 & $(0,00 ; 0,39)$ & 0,2512 & Ruim \\
\hline
\end{tabular}

Obs.: 0 s valores de $p$ menores que 0,0001 correspondem à rejeição da hipótese nula no nível de significância de $5 \%$. Classificação: excelente: kappa >0,75; bom: kappa de 0,40 a 0,75; ruim: kappa $<0,40$.

FIT, fossa infratemporal; SE, seio esfenoidal; FOS, fissura orbitária superior; SC, seio cavernoso; FCM, fossa craniana média; BPP, base do processo pterigóide; FCA, fossa craniana anterior. 
e extensão, em virtude do emprego sistemático da TC, por sua capacidade de avaliar detalhes ósseos da base do crânio ${ }^{(12,15)}$. A avaliação tomográfica da extensão tumoral deve ser baseada em um profundo conhecimento anatômico do pescoço e base do crânio, bem como de completo entendimento das vias de extensão tumoral.

Do ponto de origem na fossa pterigopalatina, adjacente à abertura do canal pterigóide, o tumor pode estender-se lateralmente ocupando a fossa pterigopalatina, fossa infratemporal e fossa temporal. Deste ponto, progride superiormente para a fissura orbitária inferior. Quando alcança este local, erode parte da grande asa do esfenóide, condicionando alargamento da fissura orbitária superior, atingindo a fossa craniana média e, finalmente, o seio cavernoso. Posteriormente, o tumor pode se estender através do canal pterigóide, erodir a asa interna do processo pterigóide, seguindo para o soalho do seio esfenóide e se desenvolver dentro deste ou crescer, inferiormente, para a fossa interpterigóidea, bem como para o espaço parafaríngeo, lateral ao hâmulo do pterigóide. Se o tumor seguir a tuba auditiva e chegar aos forames oval e redondo, conseguirá progredir para o seio cavernoso. A extensão inferior do tumor pode atingir o forame lácero e a artéria carótida interna. A extensão superior, condicionando invasão da fossa craniana anterior, através da lâmina horizontal do etmóide, raramente ocorre. Este tumor cresce lentamente e prefere locais de menor resistência, tornando pouco provável esta via de extensão ${ }^{(5,13,15-17)}$.

$\mathrm{O}$ índice de concordância interobservadores na avaliação da extensão tumoral para a fossa infratemporal foi excelente e uniforme. Isto se deve, muito provavelmente, à facilidade em se avaliar o tecido tumoral, que se apresenta hipervascularizado, facilmente diferenciado dos tecidos músculo-gordurosos presentes nesta topografia. Imagens tomográficas nos planos coronais e, principalmente, axiais apresentam boa visibilização desta invasão ${ }^{(1)}$, sendo fundamental a infusão endovenosa de meio de contraste.

O maior risco de recorrência para o envolvimento da fossa infratemporal está relacionado com a escolha de uma abordagem anterior para a ressecção tumoral, aumentando a possibilidade de ressecção incompleta. Outro fator descrito é a adesão aos tecidos moles desta região, o que torna difícil a completa separação tumoral dos tecidos adjacentes (Figura 1).

$\mathrm{O}$ índice de concordância interobservadores na avaliação da invasão da fissura orbitária superior foi bom. Este dado se deve à diferenciação entre o tecido tumo- ral, que aparece altamente vascularizado e com altos índices de atenuação, comparados com os baixos índices de atenuação do tecido gorduroso nesta localização. Outro fator a ser considerado é que qualquer alargamento da fissura orbitária superior pode ser considerado como positivo para o envolvimento ${ }^{(18)}$ (Figura 2). A possibilidade de comparação com o lado contralateral torna a avaliação mais precisa. As imagens nos planos axial e coronal são necessárias para a correta avaliação, bem como janela e nível de abertura de janela apropriados para a avaliação de tecidos com densidade de partes moles e nível e abertura de janela para a avaliação do tecido ósseo.

O crescimento tumoral passando pela fissura orbitária superior é a rota mais freqüente de invasão intracraniana ${ }^{(3,19)}$. Som e Curtin referiram que se a fissura orbitária superior encontra-se alargada, a extensão intracraniana está presente na grande maioria dos $\operatorname{casos}^{(18)}$.

$\mathrm{O}$ índice de concordância interobservadores na avaliação da extensão tumoral para o seio esfenoidal foi excelente e uniforme para os três observadores. Isto se deve à facilidade em se diferenciar o tecido tumoral, que apresenta densidade de partes moles, do ar presente no interior do seio (Figuras 2 e 4). Imagens tomográficas no plano coronal são mandatórias,
Figura 1. Tomografia computadorizada, corte axial com janela para avaliação de partes moles após a infusão endovenosa de contraste demonstrando massa na nasofaringe à esquerda, alargando a fossa pterigopalatina, deslocando anteriormente a parede posterior do antro maxilar e invadindo a fossa infratemporal. Nota-se a diferenciação entre 0 tecido tumoral altamente vascularizado e estruturas músculogordurosas desta localização.

Figura 2. Tomografia computadorizada, corte coronal com janela para avaliação de partes moles após a infusão endovenosa de contraste demonstrando massa na nasofaringe à esquerda, invadindo o seio esfenoidal, alargando e ocupando a fissura orbitária superior, estendendo-se para a fossa infratemporal.

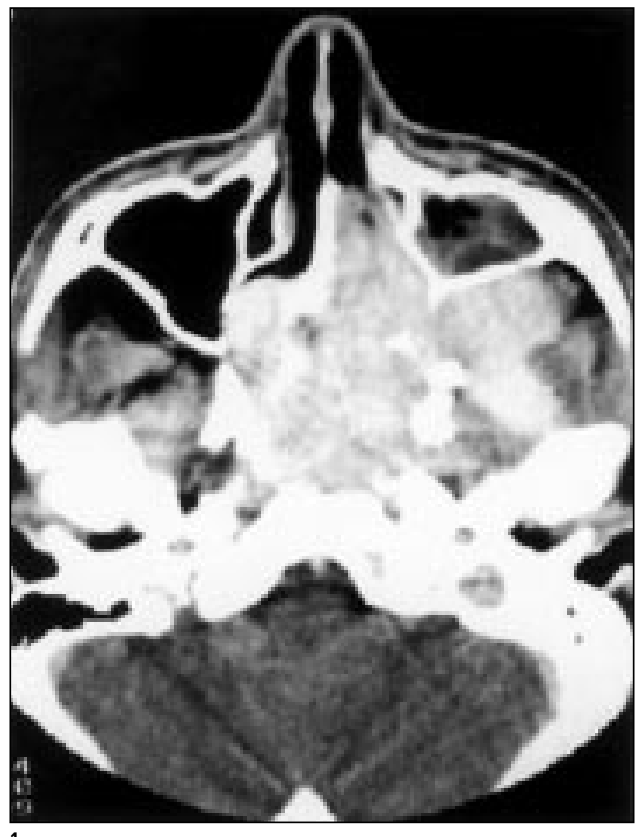

1

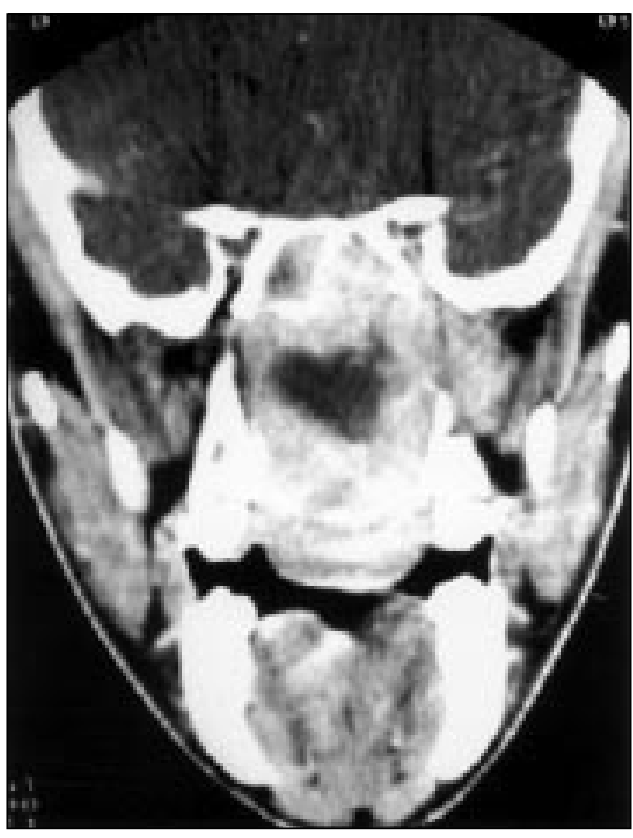

2 
devido à melhor avaliação do envolvimento do soalho do seio, avaliação esta que pode ser difícil apenas em imagens no plano axial ${ }^{(20,21)}$.

Atingindo esta localização de baixa resistência, o tumor pode crescer sem oposição, adquirindo grandes dimensões. Crescendo neste local, aumenta a possibilidade de invasão intracraniana.

O índice de concordância interobservadores na avaliação da invasão da base do processo pterigóide foi ruim. $\mathrm{O}$ fator que provavelmente explica este baixo índice é o contato tumoral com mínima erosão óssea, o que dificulta a análise, levando a erros na interpretação. Para a correta avaliação deste envolvimento, é mandatória a realização de imagens com nível e abertura de janela próprios para a avaliação dos tecidos ósseos, bem como realização de cortes finos (Figura 3 ).

O valor deste envolvimento está relacionado à grande dificuldade em se ressecar o tumor, que pode se apresentar altamente ancorado no tecido ósseo. Esta lesão dificilmente é ressecável em sua totalidade, estando as recidivas associadas a tecido tumoral remanescente. $\mathrm{Na}$ série descrita por Lloyd et al., 93\% das recorrências ocorreram com este tipo de extensão ${ }^{(12)}$.

O índice de concordância interobservadores na avaliação do seio cavernoso foi excelente. Isto se deve à possibilidade em se comparar os seios cavernosos, que devem ser simétricos. Qualquer assimetria, associada à continuidade com o tumor, pode ser considerada como envolvimento (Figura 4). A TC com infusão endovenosa de meio de contraste iodado é mandatória para a correta avaliação desta localização.

O valor desse envolvimento está relacionado à mudança no tratamento desses pacientes. Esta localização está relacionada a grande risco cirúrgico, pois qualquer abordagem do seio cavernoso coloca em risco as estruturas nervosas que o atravessam (III, IV, primeiro ramo do $\mathrm{V}$ e VI pares cranianos), os quais, se lesados, levam a oftalmoplegia e redução da acuidade visual ${ }^{(13,22)}$.

$O$ índice de concordância interobservadores na avaliação da invasão da fossa craniana anterior foi ruim.

A erosão óssea do soalho da fossa craniana anterior é bem reconhecida em imagens no plano coronal com abertura e nível de janela apropriados para a avaliação óssea, porém uma mínima invasão pode levar a uma dificuldade na avaliação. A TC com infusão endovenosa de meio de contraste iodado é mandatória para a correta avaliação desta localização, assim como a realização de imagens no plano coronal, que é o plano de eleição para o envolvimento de estruturas intracranianas e imagens utilizando-se abertura e nível de janela próprios para a avaliação óssea.
Para a ressecção, uma abordagem crânio-facial deve ser realizada, sendo muitas vezes necessária uma ação combinada entre os cirurgiões de cabeça e pescoço e os neurocirurgiões ${ }^{(23)}$.

O índice de concordância interobservadores na avaliação da invasão da fossa craniana média foi excelente. Isto se deve à possibilidade de avaliação de erosão óssea da base do crânio, alargamentos foraminais e à diferença de atenuação do tecido cerebral e do tumor, que se apresenta hipervascularizado (Figura 5). A TC com infusão endovenosa de meio de contraste iodado é mandatória para a correta avaliação desta localização, bem como a realização de imagens no plano coronal $^{(24,25)}$.

O valor deste envolvimento está relacionado à mudança na abordagem cirúrgica, porque a via de acesso deve ser combinada. O aumento do índice de recidivas tumorais está fortemente relacionado com a invasão intracraniana, sobretudo da fossa craniana média, que é a localização intracraniana mais freqüente. Extensão intracraniana ocorre de $5 \%$ a $20 \%$ dos casos e primariamente envolve a fossa craniana média ${ }^{(3,5,19)}$.

Não existem trabalhos, na literatura, que avaliem a concordância entre observadores na análise de locais de acometimento e extensão do NAFJ. O que se apresenta são estadiamentos feitos por um ou mais radiologistas.

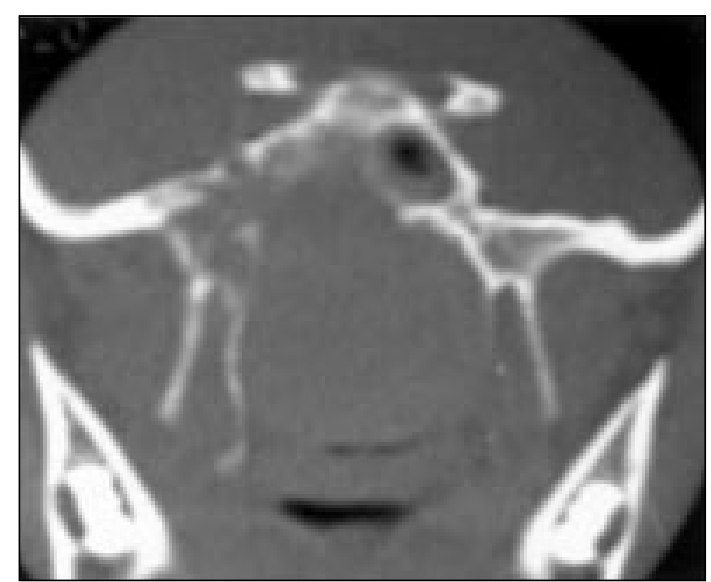

3

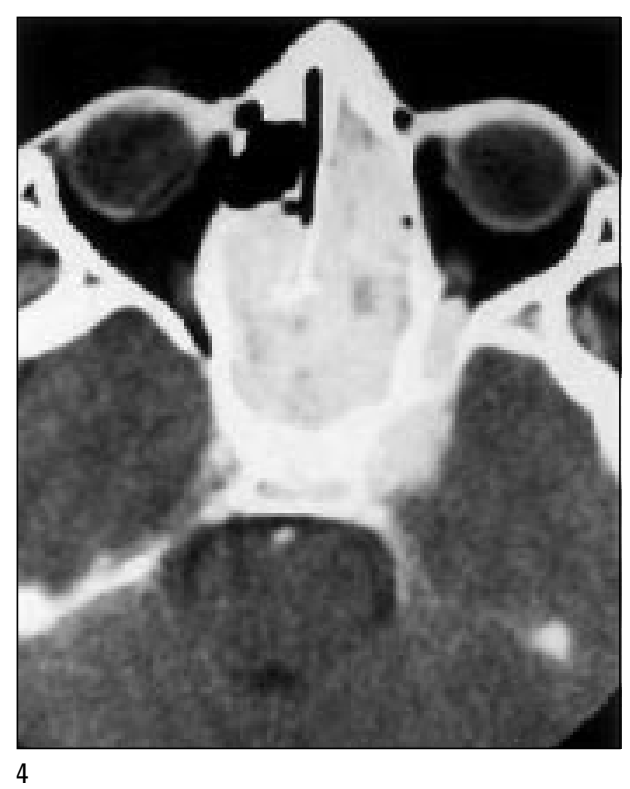

Figura 3. Tomografia computadorizada, corte coronal com janela para avaliação óssea demonstrando erosão da base do processo pterigóide à direita. Nota-se a base do processo pterigóide preservada à esquerda.

Figura 4. Tomografia computadorizada, corte axial com janela para avaliação de partes moles após a infusão endovenosa de contraste demonstrando alargamento do seio cavernoso à esquerda. Nota-se o seio cavernoso normal à direita. Existe ocupação por tecido tumoral do seio esfenoidal e células etmoidais. 


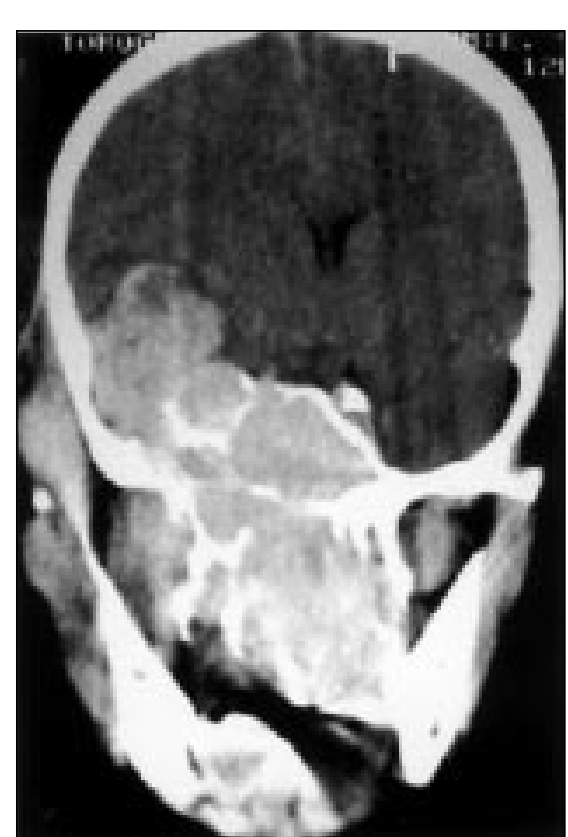

Figura 5. Tomografia computadorizada, corte coronal com janela para avaliação de partes moles após a infusão endovenosa de contras te demonstrando massa na nasofaringe à direita, estendendo-se para a fossa infratemporal, erodindo a base do processo pterigóide, invadindo 0 seio esfenoidal, alargando o seio cavernoso, estendendo-se para a fossa craniana média.

A análise da concordância entre observadores é importante, na medida em que o estadiamento pré-operatório do NAFJ é tomográfico, sendo fundamental a correta descrição dos locais de acometimento para um planejamento cirúrgico seguro.

Observa-se uma concordância que, em média, é boa para todos os locais, embora os índices de concordância para a base do processo pterigóide e fossa craniana anterior apresentem-se baixos.

\section{CONCLUSÕES}

A avaliação interobservadores é excelente e uniforme para a fossa infratemporal e seio esfenóide.

Existe, em média, boa concordância entre os observadores para a avaliação da fissura orbitária superior, seio cavernoso e fossa craniana média.

Existe, em média, baixa concordância entre os observadores para a avaliação da erosão da base do processo pterigóide e invasão da fossa craniana anterior.

\section{REFERÊNCIAS}

1. Antonelli AR, Cappiello J, Di Lorenzo D, Donajo CA, Nicolai P, Orlandini A. Diagnosis, staging, and treatment of juvenile nasopharyngeal angiofibroma (JNA). Laryngoscope 1987;97:1319-25.

2. Gupta AC, Murthy DP. Intracranial juvenile nasopharyngeal angiofibroma. Aust N Z J Surg 1997; 67:477-82.

3. Standefer J, Holt GR, Brown WE. Combined intracranial and extracranial excision of nasopharyngeal angiofibroma. Laryngoscope 1983;93:7729.

4. Davis KR. Embolization of epistaxis and juvenile nasopharyngeal angiofibromas. AJR 1987;148: 209-18

5. Bremer JW, Neel HB III, De Santo LW, Jones GC. Angiofibroma: treatment trends in 150 patients during 40 years. Laryngoscope 1986;96:1321-9.

6. Economou TS, Abemayor E, Ward PH. Juvenile nasopharyngeal angiofibroma: an update of the UCLA experience, 1960-1985. Laryngoscope 1988;98:170-5.

7. Batsakis JG. Tumors of the head and neck: clinical and pathological considerations. Baltimore: Williams \& Wilkins, 1979.

8. Shikani AH, Richtsmeier WJ. Juvenile nasopharyngeal angiofibroma tumor models. Failure of androgens to stimulate growth in nude mice and in vitro. Arch Otolaryngol Head Neck Surg 1992;118:2569.

9. Kumagami H. Estradiol, diidrotestosterone and testosterone in juvenile angiofibroma tissue. Am J Rhinol 1993;7:101-9.

10. Alvi A, Myssiorek D, Fuchs A. Extranasopharyngeal angiofibroma. J Otolaryngol 1996;25: 346-8.
11. Sessions RB, Bryan RN, Naclerio RM, Alford BR. Radiographic staging of juvenile angiofibroma. Head Neck Surg 1981;3:279-83

12. Lloyd G, Howard D, Phelps P, Cheesman A. Juvenile angiofibroma: the lessons of 20 years of modern imaging. J Laryngol Otol 1999;113:12734

13. Herman P, Lot G, Chapot R, Salvan D, Huy PTB Long-term follow-up of juvenile nasopharyngeal angiofibromas: analysis of recurrences. Laryngoscope 1999;109:140-7.

14. Lund VJ, Lloyd GAS, Howard DJ. Juvenile angiofibroma - imaging techniques in diagnosis. Rhinology 1989;27:179-85

15. Lloyd GAS, Phelps PD. Juvenile angiofibroma: imaging by magnetic resonance, CT and conventional techniques. Clin Otolaryngol 1986;11:24759.

16. Chandler JR, Goulding R, Moskowitz L, Quencer RM. Nasopharyngeal angiofibromas: staging and management. Ann Otol Rhinol Laryngol 1984; 93:322-9

17. Som PM, Cohen BA, Sacher M, Choi IS, Bryan NR. The angiomatous polyp and the angiofibroma: two different lesions. Radiology 1982;144: 329-34.

18. Som PM, Curtin HG. Head and neck imaging. 3rd ed. Saint Louis: Mosby, 1996.

19. Jafek BW, Krekorian EA, Kirsch WM, Wood RP Juvenile nasopharyngeal angiofibroma: management of intracranial extension. Head Neck Surg 1979;2:119-28.

20. Radkowski D, McGill T, Healy GB, Ohlms L, Jones DT. Angiofibroma. Changes in staging and treatment. Arch Otolaryngol Head Neck Surg 1996. 122:122-9.

21. Brooker DS, Kenny B, Gibson RG, Primrose WJ Juvenile nasopharyngeal angiofibroma in a static population: the implications of misdiagnosis. Clin Otolaryngol 1989;14:497-502.

22. Andrews JC, Fisch U, Valavanis A, Aeppli U, Makek MS. The surgical management of extensive nasopharyngeal angiofibromas with the infratemporal fossa approach. Laryngoscope 1989;99:42937

23. Krekorian EA, Kato RH. Surgical management of nasopharyngeal angiofibroma with intracranial extension. Laryngoscope 1977;87:154-64.

24. Tewfik TL, Tan AK, al Noury K, et al. Juvenile nasopharyngeal angiofibroma. J Otolaryngol 1999; 28:145-51

25. Jacobsson M, Petruson B, Svendsen P, Berthelsen B. Juvenile nasopharyngeal angiofibroma. A report of 18 cases. Acta Otolaryngol 1988;105:132-9 